\title{
ANÁLISE DE PLANOS DE GERENCIAMENTO DE RESÍDUOS DE SERVIÇOS DE SAÚDE EM HOSPITAIS EM SALVADOR, BAHIA
}

\section{ANALYSIS OF MANAGEMENT PLANS OF WASTE HEALTH SERVICES IN HOSPITALS IN SALVADOR, BAHIA}

\section{Débora Maria Oliveira Cruz Vilela}

Mestre em Saúde, Ambiente e Trabalho, Faculdade de Medicina da UFBA. Universidade Federal da Bahia / Serviço

Médico Universitário Rubens Brasil. (cruz.debora@hotmail.com)

\section{Severino Soares Agra Filho}

Doutor em Engenharia Química, UNICAMP. Universidade Federal da Bahia / Departamento de Engenharia Ambiental. (severino@ufba.br)

\section{Resumo}

O Plano de Gerenciamento de Resíduos de Serviços de Saúde (PGRSS), baseado nos princípios da Política Nacional de Resíduos Sólidos (PNRS), visa a minimizar a geração de resíduos, realizar um descarte correto no ambiente, proteger a saúde e prevenir acidentes. Diante da obrigatoriedade de sua elaboração, este artigo tem por objetivo apresentar o resultado da pesquisa realizada sobre a análise da aplicação dos PGRSS em hospitais em Salvador, Bahia. Como procedimentos metodológicos, foram realizadas pesquisas bibliográfica e documental, observação em campo e entrevistas. Os resultados permitiram constatar a fragilidade e precariedade da qualidade das informações e dos procedimentos de gerenciamento especificados nos Planos. Os principais pontos desfavoráveis encontrados nos PGRSS, como a ausência de quantificação dos RSS gerados, a segregação deficiente e o armazenamento externo, representam deficiências que põem em risco a saúde e o meio ambiente. Assim, conclui-se que os PGRSS não atendem totalmente aos propósitos estabelecidos nas legislações e normas vigentes.

Palavras-chave: Plano de Gerenciamento; Resíduos de Serviços de Saúde; Hospital

\section{Abstract}

The Health Services Waste Management Plan (PGRSS), based on the principles of the National Solid Waste Policy (PNRS), aims to minimize the generation of waste, perform a correct disposal in the environment, protect health and prevent accidents. Faced with the obligation to elaborate it, this article aims to present the results of the research carried out, on the analysis of the application of PGRSS in hospitals in Salvador, Bahia. As methodological procedures were carried out bibliographical research, documentary, field observation and interviews. The results showed the fragility and precarious quality of the information and management procedures specified in the Plans. The main unfavorable points found in the PGRSS, such as the lack of quantification of RSS generated, poor segregation and external storage, represent deficiencies that endanger health and the environment. Thus, it is concluded that the PGRSS do not fully comply with the purposes established in the laws and regulations in force.

Keywords: Management Plan; Waste Health Services; Hospital

\section{INTRODUÇÃO E FUNDAMENTAÇÃO TEÓRICA}

A grave problemática dos resíduos sólidos assume um papel de destaque e, por isso, convoca a sociedade, gestores públicos e organizações privadas a discutirem sobre o tema e a assumirem uma nova postura diante dos padrões de consumo impostos pelo modo de produção vigente. Os resíduos de serviços de saúde (RSS) fazem parte dessa problemática na medida em que apresentam potencial significativo de risco ao meio ambiente e à saúde pública, devido às suas características de patogenicidade (SCHNEIDER et al., 2004; SALOMÃO; TREVIZAN; GUNTHER, 2004; SILVA; HOPPE, 2005). Dados da Organização Mundial de Saúde (OMS) estimam que em torno de $10 \%$ a $25 \%$ dos resíduos gerados pelos prestadores de serviços de saúde são considerados infectantes, podendo provocar riscos ambientais e à saúde (WHO, 2014). 
Nesse sentido, o gerenciamento adequado dos RSS, buscando atender aos princípios e objetivos da Política Nacional de Resíduos Sólidos - PNRS, deve considerar a ordem de prioridades estabelecida pela mesma, a qual visa a não geração, redução, reutilização e reciclagem por meio de alternativas que vão desde a mudança de procedimentos e tecnologias até o retorno dos resíduos ao ciclo produtivo (BRASIL, 2010). Assim, as etapas do gerenciamento devem seguir um caminho que visem a minimizar a quantidade de resíduos antes do seu tratamento ou disposição final dos rejeitos.

As diretrizes da PNRS, consolidando as Resoluções da Agência Nacional de Vigilância Sanitária (RDC no 306, da ANVISA), bem como do Conselho Nacional de Meio Ambiente (Resolução $n^{\circ}$ 358, do CONAMA), determinam que todas as atividades de serviço de saúde estão obrigadas a elaborar e implantar o Plano de Gerenciamento dos Resíduos de Serviços de Saúde (PGRSS), documento que contempla as informações e procedimentos referentes à geração, segregação, acondicionamento, coleta, armazenamento, transporte, reciclagem, tratamento e disposição final, como também as características, classificação e volume dos resíduos de serviços de saúde gerados (BRASIL, 2004; 2005; GARCIA; ZANETTI-RAMOS, 2004).

Oliveira et al. (2013) destacam que a adoção de um PGRSS pelo Serviço de Saúde deve considerar três aspectos fundamentais, a saber: a organização do sistema de manuseio dos RSS; aspectos técnico-operacionais; e recursos humanos devidamente capacitados para 0 funcionamento do sistema. Nesse sentido, Baggio e Machado Júnior (2013) chamam atenção para a importância do PGRSS como fonte de informação para o gestor, pois a falta de informação técnica ao gerador compromete uma gestão adequada dos resíduos.

O PGRSS deve ser elaborado seguindo parâmetros técnicos, normativos e legais, baseados na análise de geração dos resíduos do estabelecimento de saúde e nos princípios de não geração e minimização da geração (BRASIL, 2004). Entretanto, não basta apenas elaborar o Plano. É papel da gestão ambiental acompanhar a sua implementação, para que os serviços geradores de RSS não negligenciem as ações relativas ao manejo dos resíduos previstas no PGRSS. Ademais, fazem-se necessárias análises periódicas e monitoramento constante por parte dos órgãos do Poder Público, a fim de fiscalizar o seu conteúdo e a efetividade do cumprimento de suas diretrizes e ações (BRASIL, 2004). Recomenda-se, portanto, que o PGRSS seja um documento "dinâmico, revisado e aprimorado diante dos avanços tecnológicos e das novas exigências legislativas" (COSTA, 2012, p. 5).

Para Nardy, Carbonari e Cunha (2007), a implantação do PGRSS busca minimizar a quantidade e gerenciar a qualidade da geração dos resíduos, especialmente aqueles que, por caráter biológico ou por suas propriedades físicas e/ou químicas, tenham alto índice de periculosidade, com consequente redução dos riscos inerentes aos resíduos.

Os principais benefícios para um estabelecimento de saúde, ao possuir um PGRSS e ter sua implantação concretizada, são: a implementação de rotinas e boas práticas de gerenciamento, redução de riscos de acidentes ocupacionais e de poluição ambiental; melhoria nas condições dos serviços de saúde oferecidos aos pacientes; e a redução da quantidade de resíduos enviados ao aterro, com consequente minimização de impactos ambientais (THOMPSON, 2012).

Nessa perspectiva, este artigo apresenta os resultados da pesquisa de mestrado da primeira autora, com o objetivo de analisar a aplicação do Plano de Gerenciamento de Resíduos de Serviços de Saúde (PGRSS) em hospitais localizados no município do Salvador, Bahia. Pretende-se caracterizar a prática adotada em termos do conteúdo e da execução dos PGRSS em distintas modalidades de hospitais, visando a avaliar a suficiência das práticas e procedimentos adotados e buscando identificar as evidências de sua efetividade.

\section{METODOLOGIA}

A pesquisa desenvolvida adotou como base metodológica uma abordagem qualitativa e caracterizou-se por ser um estudo de caso, descritivo e exploratório. Para tanto, foi procedida uma análise do conteúdo das informações e procedimentos de elaboração e de execução dos Planos de Gerenciamento de Resíduos de Serviços de Saúde (PGRSS) de quatro hospitais localizados no município do Salvador, Bahia. Nesse propósito, foi realizada tanto análise documental dos PGRSS, quanto visitas de observação e entrevistas nas unidades selecionadas. Assim, a pesquisa buscou caracterizar como estão sendo elaborados e 
executados os PGRSS, adotando a seguinte questão orientadora: Quais as evidências sobre a efetividade da elaboração e implementação do Plano de Gerenciamento de Resíduos de Serviços de Saúde para a gestão de hospitais?

Para análise do conteúdo do PGRSS, a partir da pesquisa bibliográfica procedida (SCHNEIDER et al., 2004; COSTA, 2012; OPAS, 1997; CUSSIOL, 2008; BRASIL, 2006; 2012b), estabeleceu-se a confrontação das exigências previstas na revisão de literatura, reunidas em um Referencial Teórico de Análise (RTA). Nas apreciações dos PGRSS analisados, foram consideradas as informações fornecidas e os procedimentos e suas correspondentes especificações. Para tanto, adotaram-se os critérios indicados nos Quadros 1 e 2.

Quadro 1: Critérios utilizados para analisar a qualidade das informações nos PGRSS, de acordo com o Referencial Teórico de Análise

\begin{tabular}{|cc|}
\hline & INFORMAÇõES \\
\hline Qualidade das informações & Fornecimento de dados previstos \\
\hline Informações suficientes & Fornece todos os dados e informações esperadas \\
\hline & $\begin{array}{c}\text { Incompleto no fornecimento de informações } \\
\text { esperadas, que prejudica gravemente o PGRSS }\end{array}$ \\
\cline { 2 - 3 } Informações parcialmente suficientes & $\begin{array}{c}\text { Incompleto no fornecimento de informações } \\
\text { esperadas, que prejudica o PGRSS }\end{array}$ \\
\hline Informações inexistentes & $\begin{array}{c}\text { Nenhuma informação é fornecida ou está presente no } \\
\text { PGRSS }\end{array}$ \\
\hline
\end{tabular}

Fonte: autoria própria.

A fim de diferenciar e destacar a gravidade das informações ausentes na categoria "Informações parcialmente suficientes", adotou-se como orientação as diretrizes constantes no PNRS, que versa sobre a ordem de prioridades para a gestão e gerenciamento dos resíduos sólidos, bem como as definições de PGRSS constantes nas legislações vigentes. Baseado nessas orientações, formulou-se 0 seguinte questionamento: "A presença da informação contribuiria para ações de não geração e de minimização da geração de resíduos?":

- Se sim: Informação ausente prejudica gravemente o PGRSS;

- Se não: Informação ausente prejudica o PGRSS.

Quanto aos procedimentos (Quadro 2), considerou-se os seguintes critérios:

\section{Quadro2: Critérios utilizados para analisar a adequação dos procedimentos especificados nos PGRSS, de acordo com o RTA}

\begin{tabular}{|cc|}
\hline \multicolumn{2}{|c|}{ PROCEDIMENTOS } \\
\hline Qualidade das especificações dos procedimentos & Procedimentos previstos \\
\hline Suficientemente especificados & $\begin{array}{c}\text { Procedimentos descritos estão suficientes para } \\
\text { orientar a execução do PGRSS }\end{array}$ \\
\hline Parcialmente suficientes & $\begin{array}{c}\text { Procedimentos descritos estão parcialmente } \\
\text { especificados para orientar a execução do PGRSS }\end{array}$ \\
\hline Insuficientemente especificados & $\begin{array}{c}\text { Procedimentos descritos estão inadequados ou } \\
\text { nenhum procedimento consta no PGRSS }\end{array}$ \\
\hline
\end{tabular}

Fonte: autoria própria.

Formulado como procedimento para análise e apreciação dos PGRSS dos hospitais, com as características dos aspectos e elementos constitutivos considerados fundamentais para apreciação e execução dos PGRSS, o RTA tem em seu conteúdo as expectativas de um PGRSS almejado.
Após a elaboração do RTA, foi realizada a análise documental dos PGRSS e relatórios afins dos hospitais analisados. Posteriormente, a fim de dar sustentação aos argumentos teóricos, bem como complementar as informações obtidas nas fases anteriores, procedeu-se 0 trabalho de campo com a realização de entrevistas e 
observação. A análise do PGRSS e o trabalho de campo foram realizados no período de agosto de 2014 a novembro de 2014.

Como critérios de seleção das unidades hospitalares, foram considerados três aspectos: 0 hospital deveria estar localizado no município de Salvador, na Bahia; possuir o PGRSS implementado; e aceitar participar da pesquisa. Já a inclusão dos entrevistados foi baseada nos seguintes critérios: estar envolvido na elaboração e/ou implementação do PGRSS; e aceitar participar da pesquisa. Pautado nesses critérios, selecionou-se os seguintes hospitais, que foram nomeados com pseudônimos a fim de preservar o anonimato:

a) Alfa: maternidade, de propriedade pública, que dispõe de 103 leitos e possui 509 profissionais. O espaço físico apresenta área total construída de $5.700 \mathrm{~m}^{2}$.

b) Beta: hospital geral, de propriedade pública, com capacidade instalada para 752 leitos e possui 2.100 funcionários. O espaço físico apresenta área total construída de $32.000 \mathrm{~m}^{2}$.

c) Gama: maternidade, de propriedade pública, que dispõe de 80 leitos e 731 funcionários. O espaço físico apresenta área total construída de $4.255 \mathrm{~m}^{2}$.

d) Delta: maternidade, de parceria públicoprivada, que dispõe de 253 leitos e possui 1.300 funcionários. O espaço físico apresenta área total construída de $13.000 \mathrm{~m}^{2}$.

Foi realizada uma análise documental dos Planos dos hospitais, apreciando as 9 (nove) etapas do manejo dos resíduos, além de outros 5 (cinco) itens considerados fundamentais para 0 conteúdo de um PGRSS. As etapas foram: classificação e caracterização dos resíduos gerados, segregação, identificação, acondicionamento, coleta e transporte internos, armazenamento temporário, armazenamento externo, coleta e transportes externos e tratamento e disposição final. Quanto aos itens que devem constar no Plano, são os seguintes: identificação da unidade, capacitação, indicadores, saúde ambiental e ocupacional e equipe responsável pela elaboração, implantação e implementação do PGRSS.

As entrevistas concedidas pelos responsáveis pela elaboração e implementação do PGRSS em cada hospital aconteceram no próprio local e turno de trabalho e seguiram um roteiro semiestruturado, composto por perguntas abertas relacionadas à elaboração do PGRSS e às ações do gerenciamento de resíduos de serviços de saúde (GRSS) desenvolvidas pelos hospitais. As observações em campo foram acompanhadas por uma funcionária da área de GRSS de cada hospital.

A análise da execução dos PGRSS estruturou-se com base na coleta dos dados primários, na observação, nos registros realizados no diário de campo e nas entrevistas semiestruturadas. Durante a análise dos Planos, foi estabelecida uma relação com as características do gerenciamento RSS de cada hospital, a fim de verificar se os PGRSS analisados eram realmente executados.

Para a realização da pesquisa, foram observadas as disposições da Resolução $\mathrm{n}^{\circ}$ 466/12 do Conselho Nacional de Saúde (CNS) (BRASIL, 2012a). Este artigo é parte de uma dissertação de mestrado, cujo projeto de pesquisa foi encaminhado ao Comitê de Ética em Pesquisa da Maternidade Climério de Oliveira da Universidade Federal da Bahia e aprovado sob o parecer número 691.839.

\section{RESULTADOS E DISCUSSÃO}

A partir da pesquisa bibliográfica procedida, identificaram-se diversos subsídios relevantes para o Referencial Teórico de Análise dos PGRSS e unidades analisadas, os quais foram constituídos, resumidamente, nos seguintes aspectos, elementos e etapas de elaboração dos planos:

a) Caracterização e identificação da unidade A caracterização deve especificar os dados sobre os serviços e procedimentos médicos prestados e respectivas atividades desenvolvidas, bem como a capacidade de atendimento $\mathrm{e}$ as estruturas existentes (SCHNEIDER et al., 2004; BRASIL, 2006; CUSSIOL, 2008; ZELTZER, 2004; OLIVEIRA et al., 2013). Essas informações tornam-se indispensáveis para a compreensão do real dimensionamento e caracterização dos potenciais riscos das unidades, bem como para a identificação e determinação da quantidade de resíduos gerada e, consequentemente, a elaboração de estratégias que possibilitem a minimização da geração dos resíduos.

b) Caracterização e classificação dos resíduos

Nessa etapa, devem ser especificados os tipos de resíduos gerados e suas características, visando a identificar as 
demandas e possibilidades de gerenciamento (SCHNEIDER et al., 2004). Tal informação é determinante na caracterização do diagnóstico da situação atual e, por conseguinte, na estruturação do planejamento de ações que visem à redução da geração dos resíduos (CUSSIOL, 2008).

c) Segregação dos resíduos

Nessa etapa, deve ser informado como ocorre a segregação dos resíduos, visando a identificar melhor os resíduos gerados, evitando sua mistura, facilitando as etapas subsequentes e reduzindo os riscos associados aos resíduos. A importância de tal informação está relacionada à possibilidade de redução de custos e à garantia de que as soluções para destinação final dos resíduos e disposição final dos rejeitos não sejam comprometidas (OPAS, 1997; SCHNEIDER et al., 2004).

d) Acondicionamento e identificação

No PGRSS, deve ser informado como ocorre o acondicionamento e a identificação dos resíduos para cada grupo, descrevendo os tipos de sacos utilizados, suas características e sua capacidade e recipientes e símbolos de identificação (BRASIL, 2004). Essa informação é relevante para o controle dos riscos, ao propiciar o reconhecimento dos RSS, bem como o desenvolvimento e a efetividade das demais etapas (CUSSIOL, 2008; SOUZA et al., 2011).

e) Coleta e transporte interno

Devem constar se a coleta e o transporte interno são realizados manual ou mecanicamente, os tipos de instrumentos, equipamentos e recipientes utilizados e a rota efetuada desde o acondicionamento até 0 local do armazenamento temporário, especificando horários, frequência, buscando não coincidir com horários de visitas, refeições ou distribuição de roupas (SCHNEIDER et al., 2004; SOUZA et al., 2011). Essa informação é fundamental para o transporte seguro e eficiente dos resíduos até o armazenamento externo (SOUZA et al., 2011).

f) Armazenamentos

Devem ser informados como ocorrem tanto o armazenamento interno quanto o externo dos resíduos, os quais visam, respectivamente, a agilizar a coleta dentro do estabelecimento, otimizando o deslocamento entre os pontos geradores e 0 ponto destinado à apresentação para coleta externa, e a garantir um local seguro, fora do estabelecimento para os RSS (BRASIL, 2004; 2005). Desta forma, tais informações têm significativa importância na determinação de um local que evite a contaminação do ambiente e dos trabalhadores, bem como assegure a proteção contra vetores e ações climáticas.

g) Coleta e transporte externos

Nessa fase, devem ser especificados: a rotina e frequência de coleta, a logística de movimentação dos resíduos até a disposição final, o tipo, a identificação e as características do veículo transportador (SCHNEIDER et al., 2004; CUSSIOL, 2008; OLIVEIRA et al., 2013). Essas informações são essenciais para o acompanhamento do bom desempenho e segurança da coleta e transporte externos, evitando derramamento ou exposição dos resíduos, uma vez que a instituição também é responsável por seus resíduos gerados mesmo após sua saída do estabelecimento de saúde.

h) Tratamento

Essa etapa deve especificar os métodos de tratamento utilizados, se o tratamento é interno ou externo, bem como os seus responsáveis (BRASIL, 2004; 2005; 2006). Dessa forma, torna-se uma informação indispensável para a compreensão da redução ou eliminação do risco de transmissão de doenças infecciosas, da redução ou eliminação da quantidade de agentes infecciosos, com possibilidade de contaminação de solo, ar e água (GONÇALVES et al., 2011), o que, consequentemente, contribui para a redução da quantidade de resíduos que serão dispostos no ambiente e para o aumento da vida útil do aterro.

i) Disposição final

No PGRSS, deve ser informado em que local ocorre a disposição final dos resíduos, bem como o responsável pela sua execução, o que permite ao estabelecimento gerador acompanhar como essa etapa está se desenvolvendo (BRASIL, 2006). Tal informação é relevante para a fiscalização das empresas responsáveis, o que permite ao estabelecimento verificar se a etapa está ocorrendo de forma segura, e se estão cumprindo as exigências do órgão ambiental competente, a fim de garantir a proteção e a qualidade do meio ambiente. 
j) Capacitação

Informações sobre os treinamentos realizados e quais pretende realizar, bem como o público alvo das capacitações (BRASIL, 2006). Assim, essa etapa é determinante para a melhoria geral do plano e, sobretudo, para a apreensão do conhecimento exigido na etapa de segregação. Por conseguinte, auxilia na redução da geração dos resíduos, na diminuição dos custos e acidentes de trabalho, promovendo a efetividade das ações do gerenciamento dos resíduos (ZELTZER, 2004; OLIVEIRA et al., 2013).

k) Equipe de elaboração, implantação e implementação

Deve informar os componentes e cargos da equipe que elabora e implementa o PGRSS, identificando o seu responsável técnico, a Anotação de Responsabilidade Técnica (ART) do responsável, com documentação anexa, o número do Conselho de Classe, e, caso haja empresa contratada para elaborálo, citar o nome da empresa, ART da empresa e número do Conselho de Classe. Essas informações são indispensáveis na determinação e identificação das habilidades e qualificação dos profissionais (BRASIL, 2006; 2012b; CUSSIOL, 2008; OLIVEIRA et al., 2013; ZELTZER, 2004).

I) Saúde ambiental e ocupacional

Deve especificar as responsabilidades e qualificações das equipes de Comissão de Controle de Infecção Hospitalar $(\mathrm{CCIH})$, Comissão Interna de Prevenção de Acidentes (CIPA), Serviço Especializado de Saúde e Medicina do Trabalho (SESMT), bem como a vacinação indicada para os funcionários e as providências adotadas em situação de emergência e acidentes (OPAS, 1997; COSTA, 2012; CUSSIOL, 2008). Essas informações são necessárias para a prática de medidas previstas na preservação da saúde do trabalhador e do ambiente.

m) Indicadores

Nesse item, devem estar especificados quais os indicadores utilizados que reflitam a situação, o tipo de atendimento e serviços prestados no estabelecimento de saúde (SCHNEIDER et al., 2004), bem como permitam estabelecer comparações antes e após as intervenções (BRASIL, 2006; 2012; ZELTZER, 2004). Dessa forma, torna-se uma informação importante na compreensão, avaliação e acompanhamento do desempenho das atividades propostas no Plano. O uso de indicadores é fundamental para que se disponha de um registro sistemático das informações do PGRSS.

A aplicação do Referencial Teórico de Análise gerou os resultados consolidados nos gráficos 1 a 4 . Esses resultados evidenciam as diversas deficiências dos planos analisados, sejam em relação à qualidade ou conteúdo do fornecimento de informações, como nos procedimentos previstos e adotados para cumprimento dos planos.

Em relação às informações fornecidas nos Planos, os resultados evidenciam que:

a) Nenhuma das etapas foi considerada como plenamente suficiente;

b) As etapas de caracterização das unidades, de caracterização e classificação e da segregação dos resíduos apresentam resultados com insuficiências que podem comprometer gravemente a elaboração dos PGRSS. Nessa situação, se identificam também as informações gravemente insuficientes relativas à capacitação da equipe.

c) As demais etapas foram identificadas como parcialmente suficientes na apresentação de informações que prejudicam a qualidade, mas sem comprometer gravemente 0 seu conteúdo.

d) Apenas o Hospital Gama apresentou ausência de informações consideradas gravemente prejudiciais ao PGRSS no item indicadores, e informações inexistentes no item armazenamento temporário.

As principais deficiências nas informações referem-se: à ausência de dados na caracterização e identificação da unidade como condições de funcionamento dos hospitais, bem como de Alvará Sanitário e Licença Ambiental, nos Hospitais Alfa, Beta e Gama, prejudicando a compreensão do real dimensionamento e caracterização dos potenciais riscos das unidades; à caracterização dos resíduos, sem informar todos os setores geradores e a quantidade total de resíduos gerados, o que dificulta identificar todos os resíduos a gerenciar; à falta de informações na etapa de segregação dos resíduos, como os óleos lubrificantes e eletroeletrônicos, além dos demais resíduos do grupo $\mathrm{D}$ potencialmente recicláveis; e ao não 
fornecimento de todos os dados previstos quanto à finalidade da segregação, que também almeja garantir que as soluções e destinações possíveis aos RSS não sejam comprometidas.

\section{Gráfico 1: Consolidação dos resultados do PGRSS do Hospital Alfa}

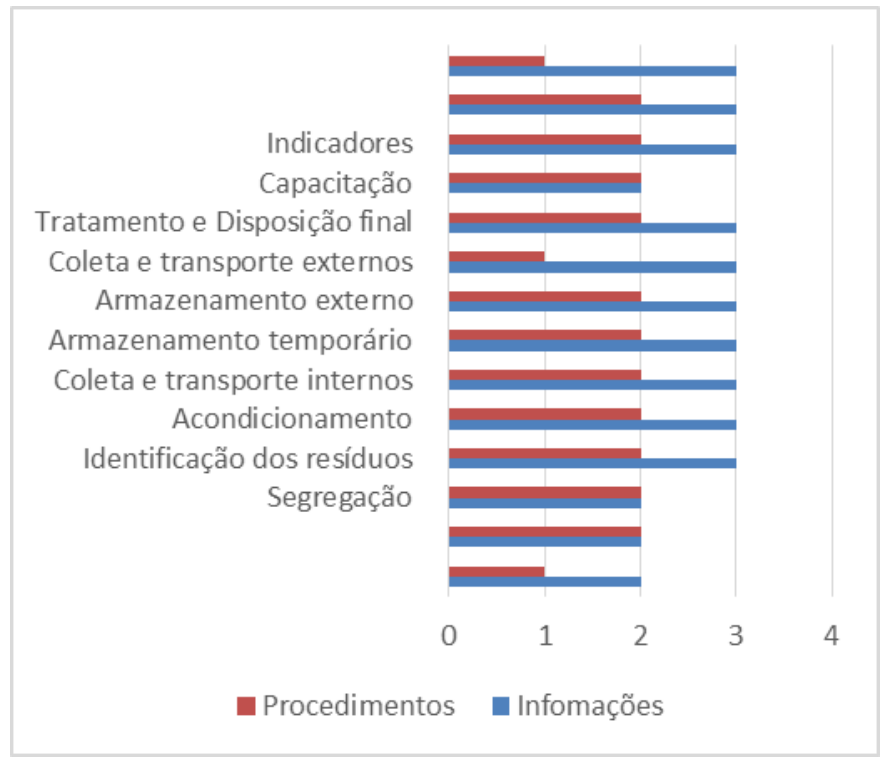

Nota: Informações:

1 - Inexistente; 2 - Parcialmente suficiente, com grave prejuízo ao PGRSS;

3 - Parcialmente suficiente.

Procedimentos: 1- Insuficiente; 2 - Parcialmente suficiente.

Fonte: autoria própria.

\section{Gráfico 2: Consolidação dos resultados do PGRSS do Hospital Beta}

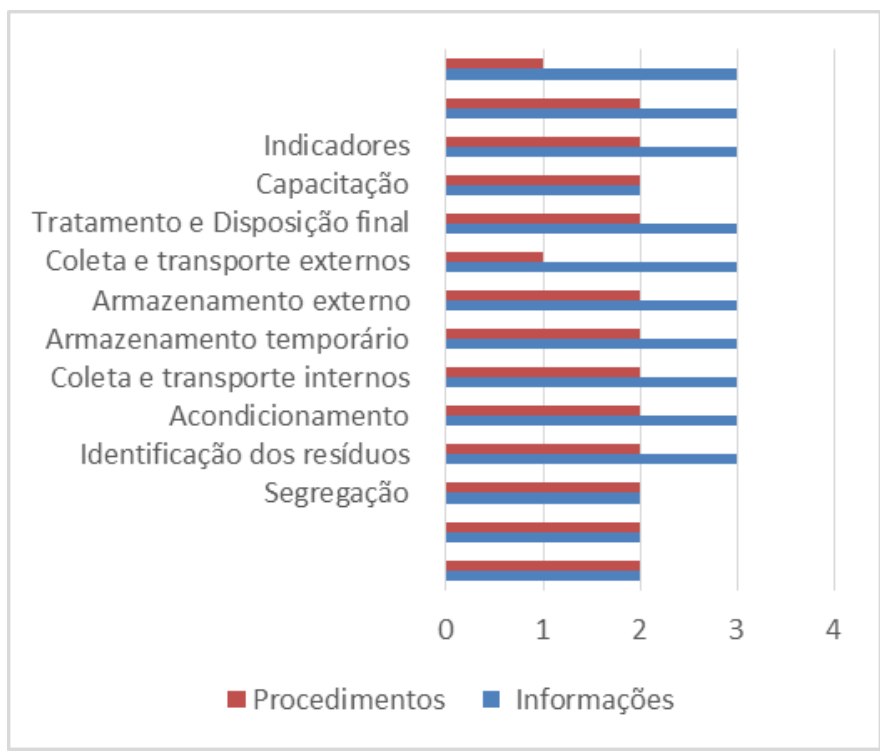

Nota: Informações:

1 - Inexistente; 2 - Parcialmente suficiente, com grave prejuízo ao PGRSS;

3 - Parcialmente suficiente.

Procedimentos: 1- Insuficiente; 2 - Parcialmente suficiente.

Fonte: autoria própria. 


\section{Gráfico 3: Consolidação dos resultados do PGRSS do Hospital Gama}

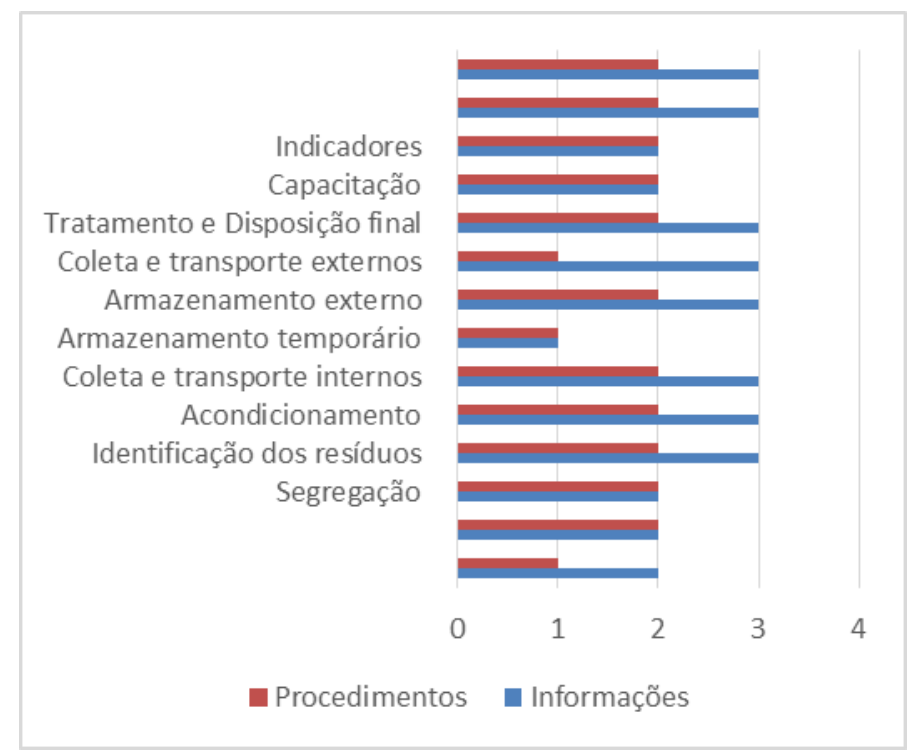

Nota: Informações:

1 - Inexistente; 2 - Parcialmente suficiente, com grave prejuízo ao PGRSS;

3 - Parcialmente suficiente.

Procedimentos: 1- Insuficiente; 2 - Parcialmente suficiente.

Fonte: autoria própria.

\section{Gráfico 4: Consolidação dos resultados do PGRSS do Hospital Delta}

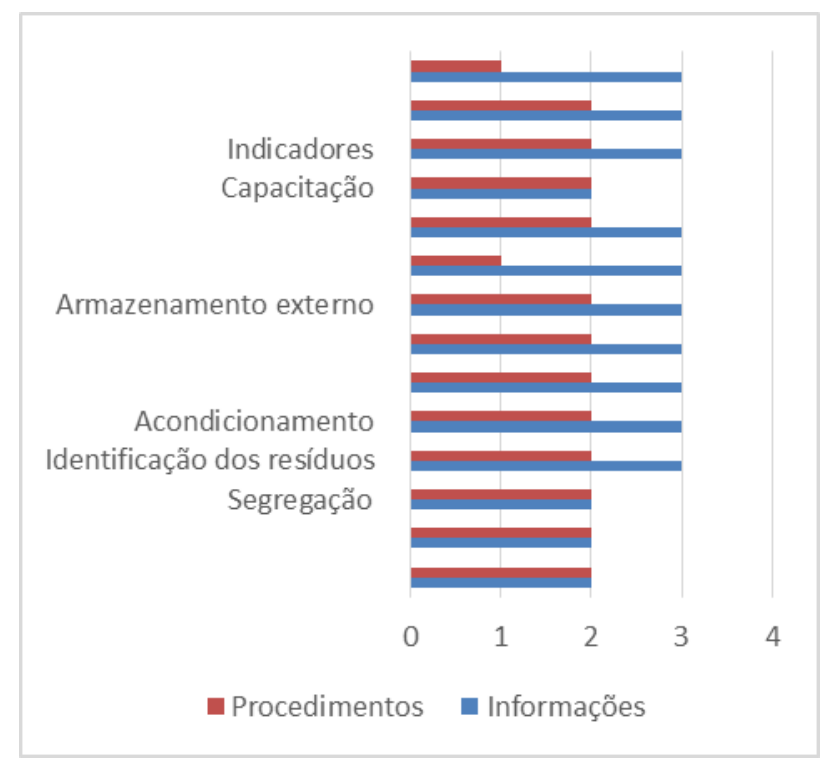

Nota: Informações:

1 - Inexistente; 2 - Parcialmente suficiente, com grave prejuízo ao PGRSS;

3 - Parcialmente suficiente.

Procedimentos: 1- Insuficiente; 2 - Parcialmente suficiente.

Fonte: autoria própria.

Nesse sentido, pode-se inferir que os planos apresentados revelam uma substancial fragilidade nas informações fornecidas, principalmente, nas etapas que são determinantes na cadeia do gerenciamento (caracterização e segregação dos resíduos). Observa-se também, sobretudo, fragilidade no atendimento da hierarquia das prioridades preconizadas nas diretrizes da PNRS. Pode-se observar, ainda, que mesmo as etapas menos complexas, como acondicionamento, 
coleta e armazenamento, não foram suficientemente atendidas. Essas deficiências evidenciam uma precariedade na elaboração dos PGRSS e sugerem um sério comprometimento da efetivação de medidas que visam a minimizar a geração dos resíduos e a estimular a sua redução para uma destinação final ou de um encaminhamento seguro aos mesmos.

Em relação à apreciação dos procedimentos previstos e adotados, podem-se observar as seguintes evidências:

a) Nenhuma unidade hospitalar apresentou plena suficiência de todos os procedimentos previstos para orientar a execução do PGRSS;

b) Houve uma significativa predominância de procedimentos parcialmente suficientes em todos os PGRSS analisados;

c) Houve uma incidência, nas etapas de identificação da unidade, quanto à caracterização, classificação e segregação dos resíduos;

d) Os PGRSS das unidades Beta e Delta apresentaram melhores resultados no que se refere à especificação dos procedimentos do que nos Hospitais Alfa e Gama;

e) O item coleta e transporte externos dos resíduos apresentou procedimentos insuficientemente especificados em todos os planos. A ausência de procedimentos descritos sobre esta etapa prejudica o acompanhamento dos resíduos por parte do gerador, mesmo após estes deixarem a unidade. A ocorrência de algum dano ambiental não isenta o gerador de suas responsabilidades;

f) Os itens caracterização e identificação da unidade, armazenamento temporário, coleta e transporte externos e equipe de elaboração, implantação e implementação do PGRSS apresentaram procedimentos insuficientemente especificados nos PGRSS.

As evidências indicadas revelam que, em termos gerais, sejam em relação às informações como aos procedimentos, os PGRSS apresentam significativas fragilidades nas etapas de caracterização e identificação das unidades, bem como quanto à caracterização, classificação e segregação dos resíduos. Essas precariedades significam que os Hospitais não identificam todas as fontes geradoras de resíduos, bem como não mantêm dados atualizados referentes à quantidade gerada dos resíduos. Isso representa um sério comprometimento na efetividade dos PGRSS, indicando que não foram elaborados seguindo um estudo prévio e eficiente de todos os resíduos gerados e de todos os locais geradores; portanto, desprovidos de consistência em sua elaboração, o que prejudica todo o planejamento requerido (SCHNEIDER et al., 2004; GONÇALVES et al., 2011).

\section{- Trabalho de campo}

Em complementação às apreciações documentais apresentadas, destacamos a seguir algumas observações obtidas nas visitas e entrevistas realizadas nos hospitais, em relação aos procedimentos praticados, sobretudo em termos de segregação e armazenamento externo dos RSS. Verificaram-se resíduos segregados em recipientes inapropriados, misturados e com diferentes classificações, o que também foi constatado em outros estudos (NÓBREGA; PIMENTEL; COSTA, 2008; OROZCO et al., 2011; ADUAN et al., 2014). Essa característica observada na segregação dos resíduos apresenta uma relação com a ausência de informações e a parcial suficiência de procedimentos especificados no item capacitação dos PGRSS analisados. Assim, como verificado nos Planos, constatou-se que nem todos os envolvidos na geração de resíduos recebem treinamento e responsabilidades para manusear adequadamente os RSS. O desconhecimento e a falta de conscientização dos funcionários quanto à segregação dos resíduos foram problemas ressaltados por todas as entrevistadas responsáveis pela implementação/concretização dos PGRSS nos hospitais analisados.

Outro aspecto observado se refere ao armazenamento externo. Durante as entrevistas e visitas de observação, ficou evidente que as características dos abrigos externos de todos os hospitais não estão totalmente adequadas e apropriadas para o armazenamento dos RSS. Porém, somente as entrevistadas dos hospitais Alfa e Beta relataram dificuldade e preocupação no armazenamento dos RSS. Durante a observação, verificou-se que o abrigo externo do Hospital Alfa (figura 1) não é adequadamente identificado e não possui porta, o que permite o acesso de pessoas não autorizadas. 
Figura 1: Abrigo e armazenamento externos dos RSS do Hospital Alfa
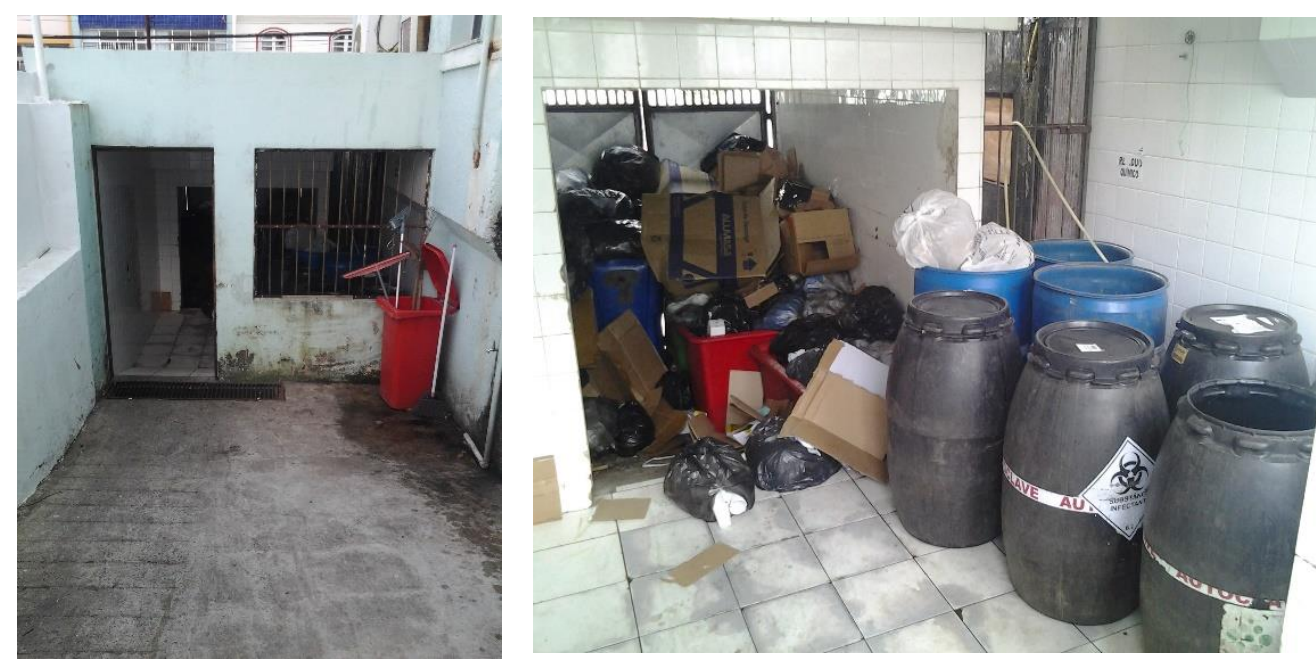

Fonte: autoria própria.

O armazenamento dos resíduos comuns é feito em sacos e caixas de papelão diretamente sobre o piso, contrariando, assim, as exigências previstas na RDC no 306/04 da ANVISA, que determina o armazenamento dos sacos dentro de contêineres (BRASIL, 2004). Os sacos contendo os resíduos biológicos, químicos e perfurocortantes são armazenados dentro de bombonas, sendo que algumas, durante a visita de observação, permaneceram abertas. Constatou-se que o abrigo externo dos resíduos do Hospital Alfa não é exclusivo, isto é, não possui ambientes fisicamente separados para receber os resíduos do Grupo A, juntamente com o Grupo E, e um ambiente para o Grupo D. Essas informações de inadequações constam no PGRSS deste Hospital, 0 que reflete 0 conhecimento da não adequação dos procedimentos por parte da gestão.

Analisando as ações de GRSS no Hospital Beta, verificaram-se as inadequadas condições do armazenamento interno de resíduos, uma vez que estes são abrigados em sacos pretos, brancos e azuis misturados em um mesmo contêiner, sendo separados somente ao chegarem ao abrigo externo. Também foram verificados papelões e galões plásticos diretamente sobre o chão do abrigo interno, indo de encontro aos procedimentos especificados no PGRSS. Quanto ao armazenamento e abrigo externos dos resíduos (figura 2), constataram-se condições precárias, corroborando, portanto, os procedimentos categorizados como parcialmente suficientes na análise do Plano.

Figura 2: Abrigo e armazenamento externos dos RSS no Hospital Beta

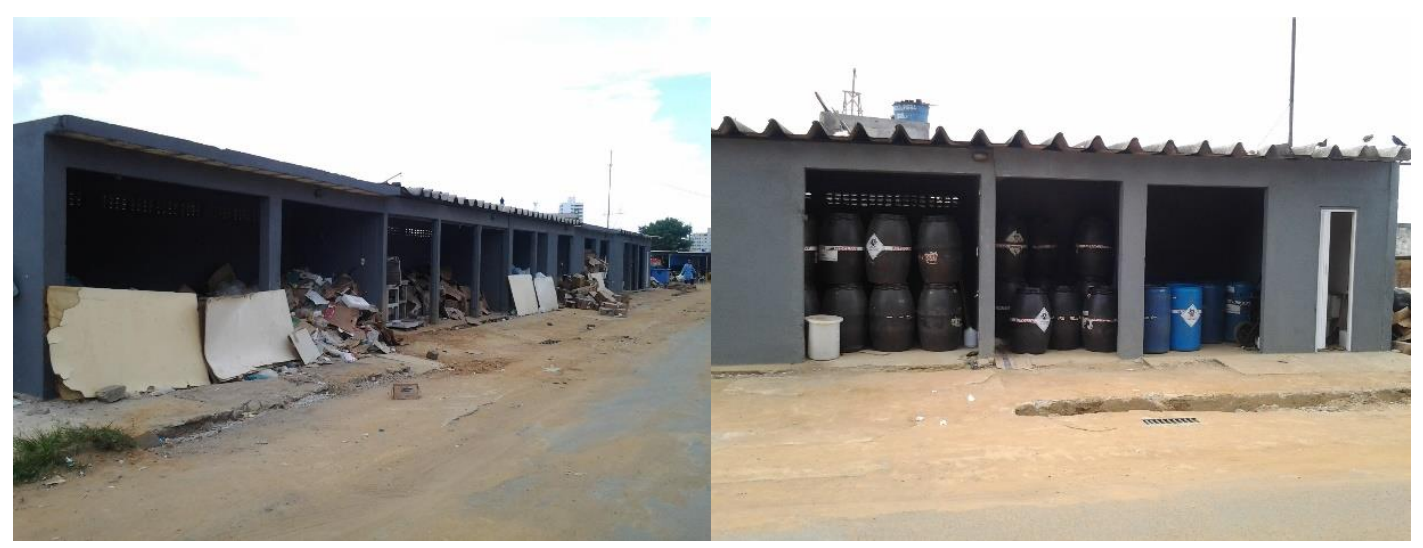

Fonte: autoria própria. 
Observa-se que o abrigo externo do Hospital Beta não possui identificação, portas e nem canaletas de escoamento de água direcionadas para rede de esgoto. Os resíduos comuns recicláveis encontram-se desorganizados, diretamente sobre o piso do abrigo, misturados a outros resíduos comuns, o que facilita a aparição de vetores e roedores; não é revestido de piso e parede de material lavável, indo de encontro ao que se preconiza na RDC n. 306/04 da ANVISA (BRASIL, 2004). Os resíduos biológicos, químicos e perfurocortantes são armazenados em bombonas fechadas em salas separadas.

No Hospital Gama, chama a atenção o odor fétido gerado pela decomposição de resíduos orgânicos e as insatisfatórias condições do armazenamento e abrigo externos dos resíduos (figura 3). $\mathrm{O}$ abrigo externo possui portas que não permitem ventilação e nem colocação de telas de proteção contra vetores. Há identificação em dois dos três boxes existentes. No box onde está identificado "lixo orgânico" é armazenado resíduo comum (papel, plástico, resíduos não orgânicos), pois, segundo a entrevistada, esta é a maior sala do abrigo e, devido à grande geração desse grupo de resíduos, é a única que comporta a quantidade diária gerada. Não há higienização diária do abrigo. Os sacos contendo os resíduos comuns (orgânico e não orgânico) são dispostos diretamente sobre o piso do abrigo que não apresenta, em um box, parede e piso revestidos de material lavável, contrariando a RDC n. 306/04 da ANVISA (BRASIL, 2004). Os resíduos biológicos, químicos e perfurocortantes são armazenados em bombonas fechadas, em sala exclusiva.

\section{Figura 3: Abrigo e armazenamento externos dos RSS do Hospital Gama}

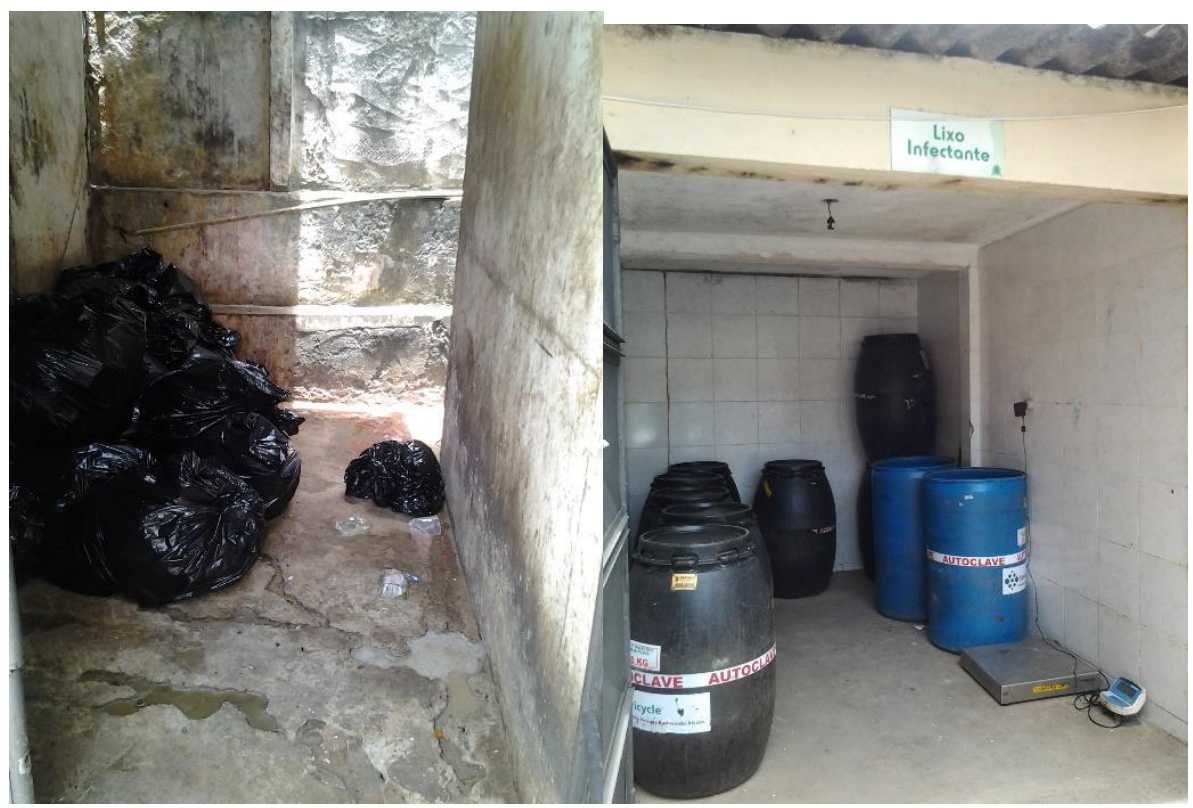

Fonte: autoria própria.

Conforme constatado in loco, o abrigo externo do Hospital Delta (Figura 4) somente apresenta identificação para o box de resíduo infectante, onde ficam armazenadas as bombonas para os resíduos biológicos, químicos e perfurocortantes. Possui portas que permitem ventilação, porém, não há telas de proteção contra vetores. As paredes e piso são constituídos de material liso e lavável. Os sacos pretos contendo os resíduos comuns são armazenados sobre o chão do abrigo e também na caixa coletora que fica ao lado do abrigo. Porém, quando a mesma se encontra com sua capacidade de armazenamento esgotada, os sacos de resíduos são deixados no chão, ao lado da caixa. Essas observações vão de encontro ao PGRSS, na medida em que o documento informa que 0 abrigo externo possui portas com proteção para vetores e é identificado. 
Figura 4: Abrigo e armazenamento externos dos RSS do Hospital Delta

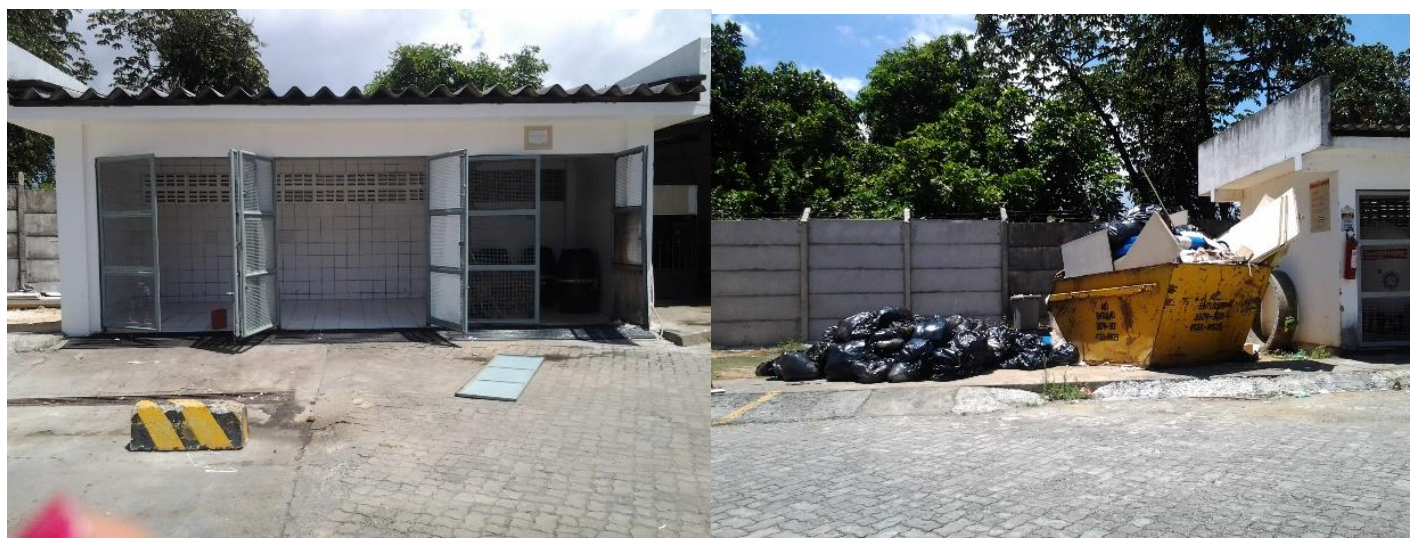

Fonte: autoria própria.

No Hospital Delta, apesar de os sacos dos resíduos no abrigo externo também permanecerem sobre 0 chão, não foram verificados resíduos e sujeiras fora dos sacos.

A situação do armazenamento dos RSS constatada em todos os hospitais indica o mau dimensionamento do número de contêineres para armazenar a quantidade de resíduos gerados nas instituições, o que aumenta a probabilidade de proliferação de vetores e roedores. Fato semelhante foi verificado em estudos realizados por Valadares (2009), Moreira (2012) e Silva, Sperling e Barros (2014).

Com relação ao tratamento dos RSS, verificou-se a necessidade de tratamento interno dos resíduos do subgrupo $\mathrm{A} 1 \mathrm{em}$ todos os Hospitais, assim como constatado em outras pesquisas (MARTINS; MAIA: BRITO, 2007; SALES et al., 2009; MARANHÃO; SOUZA; TEIXEIRA, 2013). Ademais, os resultados apontam para a necessidade de gerenciamento de efluentes, devido à presença de agentes biológicos e à alta toxicidade dos resíduos hospitalares, uma vez que essa segregação é somente realizada pelo Hospital Beta.

Quanto à destinação final dos resíduos, verificou-se nas visitas de campo que não há destinação ambientalmente adequada dos resíduos potencialmente recicláveis nos hospitais Alfa e Gama, já que os resíduos e rejeitos são dispostos em aterro sanitário. Constata-se, portanto, que o propósito do Plano de evitar o descarte de resíduos potencialmente recicláveis no aterro não está sendo devidamente cumprido. Além disso, devido à segregação deficiente, apurou-se que resíduos infectantes e perigosos também estão sendo destinados diretamente no aterro, sem o devido tratamento. Fato semelhante foi verificado em estudo realizado por Sampaio e Agra Filho (2014), no qual verificaram que uma parcela das películas de chumbo foi descartada em lixeira comum e inapropriada.

As ações de gestão ambiental praticadas nos hospitais estão ainda insuficientes. A existência de Comissão de GRSS não garantiu a satisfatoriedade da elaboração e aplicação do PGRSS. A fim de obter um bom funcionamento da comissão, é necessária uma mudança comportamental e no modo como os hospitais veem a questão ambiental. Identifica-se a necessidade de aprimoramento das ações desenvolvidas, principalmente nos hospitais Alfa, Beta e Gama, com a finalidade de garantir uma gestão mais eficiente na busca da proteção ambiental.

\section{CONCLUSÃO}

A partir dos resultados do estudo, foi possível constatar a fragilidade e precariedade da qualidade das informações e dos procedimentos de gerenciamento especificados nos PGRSS. Os principais pontos desfavoráveis encontrados nos PGRSS, como a ausência de quantificação dos RSS gerados, a segregação deficiente dos resíduos e o armazenamento externo, representam deficiências de gerenciamento, o que põe em risco tanto a saúde quanto o meio ambiente. Dessa forma, pode-se concluir que os PGRSS não atentem totalmente aos propósitos estabelecidos nas legislações e normas vigentes, caracterizando-se por apresentar condições parcialmente suficientes e com grave comprometimento das suas etapas 
determinantes, o que representa indícios de graves ameaças ambientais e à saúde, comprometendo, portanto, os princípios e objetivos da PNRS. Além das exigências legais, os PGRSS dos hospitais devem estar pautados na análise técnica da geração de resíduos do estabelecimento, com objetivo de melhor gerenciar todos os resíduos e reduzir os riscos ao meio ambiente.

Nestes termos, foi evidenciada a pouca efetividade na execução dos PGRSS analisados, os quais se restringem, no estado atual, a reduzir a destinação inapropriada de resíduos de unidade de saúde, sobretudo em relação à etapa de segregação.

Os Planos podem ser melhor elaborados se a implementação/concretização do PGRSS de hospitais for realizada por profissional e/ou equipe habilitados, que possua conhecimento técnico e atitudes proativas. Identifica-se a necessidade de aprimoramento das ações desenvolvidas, principalmente nos hospitais Alfa e Gama, a fim de garantir uma gestão mais eficiente na busca da proteção e preservação ambiental. Ademais, faz-se preciso uma mudança comportamental de todos os envolvidos no GRSS, a fim de que as questões ambientais estejam inseridas nas ações cotidianas dos hospitais. Para tanto, é prioritário que os hospitais invistam em capacitações permanentes de todos os envolvidos na geração e no gerenciamento de resíduos. Essas ações devem objetivar a mudança comportamental, visando a instituir na unidade uma cultura de sustentabilidade voltada para a redução de risco e preservação do meio ambiente.

A análise procedida sugere que as fragilidades constantes nos PGRSS, embora estes tenham sido um mecanismo indutor importante no gerenciamento dos resíduos dos serviços de saúde e evitado que estes sejam destinados inadequadamente aos aterros, a sua aplicação está ainda muito aquém das necessidades preconizadas pela PNRS. Aos órgãos fiscalizadores e licenciadores, cabe uma maior fiscalização dos PGRSS que estão sendo aplicados nos hospitais, a fim de controlar suas ações e diretrizes e, assim, garantir ou minimizar impactos ambientais e à saúde púbica.

\section{REFERÊNCIAS}

ADUAN, S.A.; BRAGA, F.S.; ZANDONADE, E.; SALLES, D.; CUSSIOL, N.A.M.; LANGE, L.C. Avaliação dos resíduos de serviços de saúde do Grupo
A em hospitais de Vitória (ES). Brasil. Revista de Eng. Sanit. Ambiental, v. 19, n. 2 abr./jun, p. 133-141, 2014. Disponível em: $<$ http://www.scielo.br/pdf/esa/v19n2/1413-4152-esa-1902-00133.pdf>. Acesso em: 22 fev. 2015.

BAGGIO, J. C.; MACHADO JÚNIOR, C. Importância do Plano de Gerenciamento de Resíduos de Serviço de Saúde. In: SIMPÓSIO DE ADMINISTRAÇÃO DA PRODUÇÃO, LOGÍSTICA E OPERAÇÕES INTERNACIONAIS, XVI, 2013, São Paulo. Anais... São Paulo-SP: SIMPOI, 2013.

BRASIL. Agência Nacional de Vigilância Sanitária. ANVISA. RDC no 306, de 7 de dezembro de 2004.Regulamento Técnico para o Gerenciamento de Resíduos de Serviços de Saúde. Brasília. 2004.

Congresso Nacional. Lei n.․ 12.305 de 02 de agosto de 2010. Institui a Política Nacional de Resíduos Sólidos; altera a Lei no 9.605, de 12 de fevereiro de 1998; e dá outras providências. Brasília. 2010.

Conselho Nacional de Meio Ambiente. CONAMA. Resolução n. 358, de 29 de abril de 2005. Define sobre o tratamento e a disposição final dos resíduos dos serviços de saúde. Brasília. 2005.

Ministério da Saúde. Agência Nacional Vigilância Sanitária. ANVISA. Manual de Gerenciamento de Resíduos de Serviços de Saúde. Brasília: Ministério da Saúde, 2006.

Ministério da Saúde. Conselho Nacional de Saúde. Resolução n. 466, de 12 de dezembro de 2012. Aprova diretrizes e normas regulamentadoras de pesquisas envolvendo seres humanos. Brasília, Diário Oficial da União, 12 dez. 2012a.

Ministério da Saúde. Secretaria de Atenção à Saúde. Departamento de Atenção Especializada. Manual para elaboração do plano de gerenciamento de resíduos dos serviços de hematologia e hemoterapia. Brasília: Editora do Ministério da Saúde, 2012b.

COSTA, E. C. L. Manejo de resíduos de serviços de saúde: manual básico de procedimentos. Brasília: Câmara dos Deputados, Edições Câmara, 2012.

CUSSIOL, N. A. M. Manual do Gerenciamento de Resíduos de Serviço de Saúde. Belo Horizonte: Fundação Estadual do Meio Ambiente, 2008.

GARCIA, L. P.; ZANETTI-RAMOS, B. G. Gerenciamento dos resíduos de serviços: uma questão de biossegurança. Cad. Saúde Pública, v. 20, n. 3, p. 744-752, 2004.

GONÇALVES, E.M.N.; SANTOS, C. B.; BADARÓ, M. L. 
S.; FARIA, V. A.; RODRIGUES, E.; MENDES, M. E.; SUMITA, N. M. Modelo de implantação de Plano de gerenciamento de resíduos no laboratório clínico. J. Bras. Patol. Med. Lab., v. 47, n. 3, p. 249-255, 2011. Disponível em: $<$ http://www.scielo.br/pdf/jbpml/v47n3/v47n3a08>.

Acesso em: 04 set. 2014.

MARANHÃO, R.A.; SOUZA, M.T.S.; TEIXEIRA, C.E. Gestão de resíduos de serviços de saúde: um estudo de caso em um posto médico da Marinha do Brasil. In: SEMINÁRIOS EM ADMINISTRAÇÃO - SEMEAD, XVI, 2013, São Paulo. Anais... São Paulo-SP: FEAUSP, 2013.

MARTINS, L.M.; MAIA, J.C.; BRITO, J.S. Análise do gerenciamento dos resíduos dos serviços de saúde em um hospital público da cidade de Teresina - PI. In: CONGRESSO DE PESQUISA E INOVAÇÃO DA REDE NORTE NORDESTE DE EDUCAÇÃO TECNOLÓGICA, II, 2007, João Pessoa. Anais... João Pessoa-PB: CEFET-PB 2007.

MOREIRA, A.M.M. Gerenciamento de resíduos de serviços de saúde. Um desafio para unidades básicas de saúde. 2012. 199 f. Dissertação (Mestrado em Ciências) - Faculdade de Saúde Pública, Universidade de São Paulo, São Paulo, 2012.

NARDY, M.B.C; CARBONARI, A.; CUNHA, M.E.G. Elaboração e implantação de um plano de gerenciamento de resíduos de serviços de saúde (PGRSS) para laboratórios de ensino. Revista de Ciências Biológicas e Saúde, v. 2, n. 2, p. 100-108, 2007.

NÓBREGA, C.C.; PIMENTEL, C.H.L; COSTA, M.D. Avaliação sobre o gerenciamento de resíduos sólidos de serviço de saúde nos hospitais de João Pessoa/Paraíba/Brasil. In: SIMPÓSIO IBEROAMERICANO DE INGENIERÍA DE RESÍDUO, I, 2008, Castellón. Anais... Castellón: REDISA, 2008.

OLIVEIRA, C.R.D.A.R.; PANDOLFO, A.; MARTINS, M.S.; GOMES, A.P; PANDOLFO, L.M.; TAGLIARI, L.D. Proposta de um plano de gerenciamento dos resíduos de serviço de saúde nos estabelecimentos assistenciais de saúde de Guaporé/RS. Revista CIATEC - UPF, v. 5, n. 1, p. 1-11, 2013.

OPAS, ORGANIZAÇÃO PAN-AMERICANA DA SAÚDE. Guia para manejo interno de resíduos sólidos em estabelecimentos de saúde. Tradução de Carol Castilho Arguello. Brasília, 1997. 64p.

OROZCO, M.M.D; VIEIRA, P.C; TRINDADE, A.M.F. Avaliação do desempenho do gerenciamento dos resíduos de serviços de saúde gerados em um hospital do município de Congonhas-MG. In: CONGRESSO BRASILEIRO DE ENGENHARIA SANITÁRIA E
AMBIENTAL, 26., 2011, Rio de Janeiro. Anais... Rio de Janeiro-RJ: ABES, 2011.

SALES C. C. L.; SPOLTI, G. P.; LOPES, M. S. B.; LOPES, D. F. Gerenciamento dos resíduos sólidos dos serviços de saúde: aspectos do manejo interno no município de Marituba, Pará, Brasil. Ciência \& Saúde Coletiva, v. 14, n. 6, p. 2231-2238, 2009.

SALOMÃO, I.S.; TREVIZAN, S.D.P.; GUNTHER, W.M.R. Segregação de resíduos de serviços de saúde em centros cirúrgicos. Revista de Engenharia Sanitária e Ambiental, v. 9, n. 2, p. 108-111, 2004.

SAMPAIO, L.L; AGRA FILHO, S.S. Gerenciamento de resíduos de películas de chumbo de serviços odontológicos em Salvador, Bahia. Revista Eletrônica de Gestão e Tecnologias Ambientais (GESTA), v. 2, n. 1, p.163-171, 2014

SCHNEIDER, V.E.; EMMERICH, R.C.; DUARTE, V.C.; ORLANDIN, S.M. Manual de gerenciamento de resíduos de serviços de saúde. 2 ed. Caxias do Sul, RS: Educs, 2004. 319 p.

SILVA, C.E; HOPPE, A.E. Diagnóstico dos resíduos de serviços de saúde no interior do Rio Grande do Sul. Revista de Engenharia Sanitária Ambiental, v. 10, n. 2, p. 146-151, abr./jun. 2005. Disponível em: http://www.scielo.br/pdf/esa/v10n2/a08v10n2. Acesso em: 06 nov. 2014.

SILVA, D.F.; SPERLING, E.V.; BARROS, T.V. Avaliação do gerenciamento dos resíduos de serviços de saúde em municípios da região metropolitana de Belo Horizonte (Brasil.). Revista de Eng. Sanit. Ambiental, v. 19, n. 3, p. 251-262, jul./set. 2014. Disponível em: $<$ http://www.scielo.br/pdf/esa/v19n2/1413-4152-esa-1902-00133.pdf>. Acesso em: 22 fev. 2015.

SOUZA, A.J; PINHEIRO, B. P.J; SILVA, M.S; SOUZA, F.P. Análise do sistema de gerenciamento dos resíduos de serviços de saúde: um estudo de caso no hospital "X"no município de Campos dos Goytacazes, RJ. Perspectivas Online: Exatas \& Eng., Campos dos Goytacazes, v. 2, n. 1, p. 74-84, 2011.

THOMPSON, M.D.C.R. Plano de gerenciamento de resíduos de serviços de saúde: proposta de modelo para um hospital no Município do Panamá, República do Panamá. 2012.189 f. Dissertação (Mestrado em Ciências) - Faculdade de Saúde Pública, Universidade de São Paulo, São Paulo, 2012.

VALADARES, C.M. Gerenciamento de resíduos de serviços de saúde: estudo em hospitais da região dos Inconfidentes. 2009. 147 f. Dissertação (Mestrado em Engenharia Ambiental) - Universidade Federal de Ouro Preto, MG, 2009. 
WHO. World Health Organizacion 2014, Safe management of wastes from health-care activities / edited by Y. Net. Chartier et al. 2nd ed. Disponível em: $<$ http://www.who.int/water sanitation health/medicalwa ste/wastemanag/en/>. Acesso em: 28 ago. 2014.
ZELTZER, R. Implementando o PGRSS (Plano de Gerenciamento de Resíduos de Serviços de Saúde). Net. Rio de Janeiro: Universidade Federal Fluminense, $2004 . \quad$ Disponível em: $<$ http://www.newslab.com.br/ed anteriores/64/index.ht ml> Acesso em: 22 jan. 2015. 